\title{
An Improved Laguerre-Samuelson Inequality of Chebyshev-Markov Type
}

\author{
Werner Hürlimann \\ Swiss Mathematical Society, University of Fribourg, 1700 Fribourg, Switzerland \\ Correspondence should be addressed to Werner Hürlimann; whurlimann@bluewin.ch
}

Received 2 May 2014; Accepted 29 July 2014; Published 12 August 2014

Academic Editor: Cheng-Hong Yang

Copyright (C) 2014 Werner Hürlimann. This is an open access article distributed under the Creative Commons Attribution License, which permits unrestricted use, distribution, and reproduction in any medium, provided the original work is properly cited.

The Chebyshev-Markov extremal distributions by known moments to order four are used to improve the Laguerre-Samuelson inequality for finite real sequences. In general, the refined bound depends not only on the sample size but also on the sample skewness and kurtosis. Numerical illustrations suggest that the refined inequality can almost be attained for randomly distributed completely symmetric sequences from a Cauchy distribution.

\section{Introduction}

Let $x_{1}, x_{2}, \ldots, x_{n}$ be $n$ real numbers with first and second order moments $s_{k}=(1 / n) \sum_{i=1}^{n} x_{i}^{k}, k=1,2$. The LaguerreSamuelson inequality (see Jensen and Styan [1] and Samuelson [2]) asserts that for a sample of size $n$ no observation lies more than $\sqrt{n-1}$ standard deviation away from the arithmetic mean; that is,

$$
\left|x_{i}-\mu\right| \leq \sqrt{n-1} \cdot \sigma, \quad i=1, \ldots, n, \mu=s_{1}, \sigma=\sqrt{s_{2}-s_{1}^{2}} .
$$

Experiments with random samples generated from various distributions on the real line suggest that there is considerable room for improvement if one takes higher order moments $s_{k}=(1 / n) \sum_{i=1}^{n} x_{i}^{k}, k \geq 3$, into account. In the present note, we demonstrate that this can be done using the socalled Chebyshev-Markov extremal distributions based on the moments of order three and four or equivalently on the (sample) knowledge of the skewness and (excess) kurtosis of a real sequence. The latter quantities are denoted and defined by

$$
\begin{aligned}
S & =\frac{(1 / n) \sum_{i=1}^{n}\left(x_{i}-\mu\right)^{3}}{\sigma^{3}}=\frac{s_{3}-3 s_{1} s_{2}+2 s_{1}^{3}}{\sigma^{3}}, \\
K & =\frac{(1 / n) \sum_{i=1}^{n}\left(x_{i}-s_{1}\right)^{4}}{\sigma^{4}}-3=\frac{s_{4}-4 s_{1} s_{3}+6 s_{1}^{2} s_{2}-3 s_{1}^{4}}{\sigma^{4}}-3 .
\end{aligned}
$$

For example, if $x_{1}, x_{2}, \ldots, x_{n}$ is a "symmetric normal" real sequence with vanishing skewness and kurtosis $S=K=0$, then the following improvement holds (see Example 5):

$$
\left|x_{i}-\mu\right| \leq \sqrt[4]{2 n-3} \cdot \sigma, \quad i=1, \ldots, n \text {. }
$$

Since the bounds in (1) can be attained, one might argue that (3) is not a genuine improvement because it depends on the property of a sequence to be "symmetric normal." However, this objection cannot be made if one states improved general bounds of the type

$$
\left|x_{i}-\mu\right| \leq C(S, K, n) \cdot \sigma, \quad i=1, \ldots, n
$$

with some analytical function $C(S, K, n)$ depending on all feasible values of $(S, K)$ and the sample size $n$. According to Arnold and Balakrishnan [3] the idea of using probability inequalities to derive (1) goes back (at least) to Smith [4] (see Jensen and Styan [1], Section 2.7). The derivation is very simple. Indeed, consider the discrete uniform random variable $X$ defined by

$$
P\left(X=\frac{x_{1}-\mu}{\sigma}\right)=\frac{1}{n}, \quad i=1, \ldots, n .
$$


Clearly, $X$ is a standard random variable, which therefore satisfies the Chebyshev-Markov inequalities (also called Cantelli inequalities):

$$
\begin{aligned}
& P(X \leq x) \leq \frac{1}{1+x^{2}}, \quad x \leq 0, \\
& P(X \leq x) \geq \frac{x^{2}}{1+x^{2}}, \quad x \geq 0 .
\end{aligned}
$$

Substituting $x=\left(x_{\min }-\mu\right) / \sigma<0$ into the first inequality and $x=\left(x_{\max }-\mu\right) / \sigma>0$ into the second inequality, one gets through combination the Laguerre-Samuelson bound (1). Along the same line of proof, we derive in Section 3 a refinement of the type (4) by considering the generalized Chebyshev-Markov inequalities by known skewness and kurtosis, which is recalled in preliminary Section 2 . The result is illustrated for some sequences of symmetric type. We observe that the new bounds are sometimes rather tight. Symmetric sequences from a Cauchy distribution, whose moments do not exist, generate examples for this phenomenon.

\section{The Chebyshev-Markov Extremal Distributions by Known Skewness and Kurtosis}

Given a partial order between random variables and some class of random variables, it is possible to construct extremal random variables with respect to this partial order, which provide useful information about extreme situations in probabilistic modeling. For example, the classical ChebyshevMarkov inequalities yield the extremal random variables with respect to the usual stochastic order for random variables with a given range and moments known up to a fixed order. Extremal random variables with respect to the increasing convex and other orderings are of similar general interest. A modern account of this topic is found in Hürlimann [5], Chap. IV.

In the following, a capital letter $X$ denotes a random variable with distribution function $F_{X}(x)=P(X \leq x)$ and survival function $\bar{F}_{X}(x)=1-F_{X}(x)$. The random variable $X$ is said to precede $Y$ in stochastic order, a relation written as $X \leq_{\text {st }} Y$, if $\bar{F}_{X}(x) \leq \bar{F}_{Y}(x)$ for all $x$ in the common support of $X$ and $Y$. The class of all random variables with given support $[A, B],-\infty \leq A<B \leq \infty$, and known moments $\mu_{1}, \mu_{2}, \ldots, \mu_{n}$ is denoted by $D_{n}\left([A, B] ; \mu_{1}, \mu_{2}, \ldots, \mu_{n}\right)$ or simply $D_{n}$ in case the context is clear. For each fixed $n=2,3$, $4, \ldots$, denote by $F_{\text {st,max }}^{(n)}(x), F_{\text {st,min }}^{(n)}(x)$ the Chebyshev-Markov extremal distributions, which are solutions of the extremal moment problems

$$
F_{\text {st } \text { max }}^{(n)}(x)=\min _{X \in D_{n}}\left\{F_{X}(x)\right\}, \quad F_{\text {st, min }}^{(n)}(x)=\max _{X \in D_{n}}\left\{F_{X}(x)\right\} .
$$

Random variables with distributions $F_{\text {st,max }}^{(n)}(x), F_{\text {st,min }}^{(n)}(x)$ are denoted by $X_{\mathrm{st}, \max }^{(n)}, X_{\mathrm{st}, \min }^{(n)}$, and one has $X_{\mathrm{st}, \min }^{(n)} \leq_{\mathrm{st}} X \leq_{\mathrm{st}} X_{\mathrm{st}, \max }^{(n)}$ for all $X \in D_{n}$. For example, if $D_{2}=D_{2}\left((-\infty, \infty) ; \mu_{1}=0\right.$, $\left.\mu_{2}=1\right)$ is the space of all standard random variables, then one has (e.g., Hürlimann [5], Chap. III, Table 4.1)

$$
\begin{aligned}
& F_{\text {st,min }}^{(2)}(x)= \begin{cases}0, & x \leq 0 \\
\frac{x^{2}}{1+x^{2}}, & x \geq 0,\end{cases} \\
& F_{\text {st,max }}^{(2)}(x)= \begin{cases}\frac{1}{1+x^{2}}, & x \leq 0 \\
1, & x \geq 0,\end{cases}
\end{aligned}
$$

which in particular includes inequalities (6).

In general, to construct the stochastic ordered extremal distributions (7), it is necessary to solve the optimization problems $\max _{X \in D_{n}}\left\{E\left[I_{[x, \infty)}(X)\right]\right\}\left(\min _{X \in D_{n}}\left\{E\left[I_{[x, \infty)}(X)\right]\right\}\right)$, where $I_{[x, \infty)}(z)$ is the Heaviside indicator function, defined to be 0 if $z<x$ and 1 otherwise. It belongs to the class of extremal moment problems $\max _{X \in D_{n}}\{E[f(X)]\}\left(\min _{X \in D_{n}}\{E[f(X)]\}\right)$, where $f(x)$ is a piecewise linear function and which have been extensively studied in Hürlimann [5-7]. A general approach to solve these problems is the well-known polynomial majorant (minorant) method. It consists to find a polynomial $q(x) \geq f(x)(q(x) \leq f(x))$ of degree less than or equal to $n$ and to construct a finite atomic random variable $Z \in D_{n}$ such that all atoms of $f(Z)$ are simultaneously atoms of $q(Z)$. Indeed, suppose $q(x)$ and $Z$ have been found such that $\operatorname{Pr}(q(Z)=f(Z))=1$ and $q(x) \geq f(x)(q(x) \leq f(x))$ for all $x \in[A, B]$. Then the expected value $E[q(Z)]=$ $E[f(Z)]$ depends only on the first moments $\mu_{1}, \ldots, \mu_{n}$, and thus necessarily $Z$ maximizes (minimizes) $E[f(X)]$ over all $X \in D_{n}$.

For the present purpose, it suffices to restrict the attention to the construction of $F_{\text {st,max }}^{(4)}(x), F_{\text {st,min }}^{(4)}(x)$ over the space $D_{4}$ of all standard random variables defined on the real line $(-\infty, \infty)$ with known skewness and kurtosis pair $(S, K)$. Recall that for $X \in D_{4}$ one has $S=E\left[X^{3}\right], K=E\left[X^{4}\right]-3$. The required main result is found in the Appendix of Hürlimann [8]. First of all, the parameters must satisfy the following well-known inequality between skewness and kurtosis (e.g., Pearson [9], Wilkins [10], and Guiard [11]):

$$
\Delta=K-S^{2}+2 \geq 0
$$

Second, the extremal bounds are attained at standard triatomic random variables $Z_{x} \in D_{4}$ such that $\operatorname{Pr}\left(q_{x}\left(Z_{x}\right)=\right.$ $\left.I_{[x, \infty)}\left(Z_{x}\right)\right)=1$ for some quartic polynomial $q_{z}(x)$, and one has

$$
\begin{gathered}
F_{\mathrm{st}, \text { max }}^{(n)}(x)=1-E\left[I_{[x, \infty)}\left(Z_{x}\right)\right], \\
F_{\mathrm{st} \text {,min }}^{(n)}(x)=E\left[I_{(-\infty, x]}\left(Z_{x}\right)\right] .
\end{gathered}
$$

To describe the extremal supports of $Z_{x} \in D_{4}$ and the associated probabilities one needs the following characterization result.

Proposition 1 (characterization of standard triatomic random variables on $(-\infty, \infty)$ with skewness $S$ and kurtosis $K)$. Suppose that $\Delta=K-S^{2}+2 \geq 0$. The support $\left\{x_{1}, x_{2}, x_{3}\right\}$, 
$x_{1}<x_{2}<x_{3}$, and probabilities $\left\{p_{1}, p_{2}, p_{3}\right\}$ of a triatomic random variable $X \in D_{4}$ are uniquely determined as follows:

$$
\begin{gathered}
x_{1}=x \in(-\infty, c], \quad x_{2}=\varphi(x, \psi(x)) \in[c, \bar{c}], \\
x_{3}=\psi(x) \in[\bar{c}, \infty), \\
p_{i}=p\left(x_{i}\right), \quad i=1,2,3,
\end{gathered}
$$

where $c=(1 / 2)\left(S-\sqrt{4+S^{2}}\right), \bar{c}=-c^{-1}=(1 / 2)\left(S+\sqrt{4+S^{2}}\right)$, and the functions $\varphi(u, v), \psi(u)$, and $p(u)$ are defined by

$$
\begin{gathered}
\varphi(u, v)=\frac{S-u-v}{1+u v}, \\
\psi(u)=\frac{1}{2}\left(\frac{A(u)-\sqrt{A(u)^{2}+4 q(u) B(u)}}{q(u)}\right), \\
A(u)=S q(u)+\Delta u, \quad B(u)=\Delta+q(u), \\
q(u)=1+S u-u^{2}, \\
p(u)=\frac{\Delta}{q(u)^{2}+\Delta\left(1+u^{2}\right)} .
\end{gathered}
$$

Proof. Consult Hürlimann [8], proof of Proposition II.2.

The Chebyshev-Markov extremal distributions over the space $D_{4}$ are determined as follows.

Theorem 2. Under the assumption $\Delta=K-S^{2}+2 \geq 0$ and in the notations of Proposition 1, the distribution functions of the Chebyshev-Markov ordered extremal random variables $X_{s t, \max }^{(4)}, X_{s t, \min }^{(4)}$ for the set $D_{4}$ are described in Table 1.

Proof. Consult Hürlimann [8], proof of Theorem III.2.

\section{A Refinement of the \\ Laguerre-Samuelson Inequality}

Let $x_{1}, x_{2}, \ldots, x_{n}$ be $n$ real numbers with first four order moments $s_{k}=(1 / n) \sum_{i=1}^{n} x_{i}^{k}, k=1,2,3,4$. The mean and standard deviation are $\mu=s_{1}, \sigma=\sqrt{s_{2}-s_{1}^{2}}$. The skewness and (excess) kurtosis are denoted by $S, K$ and defined in (2). As in Section 1, consider the discrete uniform random variable $X$ defined by $P\left(X=\left(x_{1}-\mu\right) / \sigma\right)=1 / n, i=1, \ldots, n$. Since $X$ is a standard random variable with skewness and kurtosis $S, K$, it satisfies by Theorem 2 the Chebyshev-Markov inequalities

$$
\begin{gathered}
P(X \leq x) \leq p(x), \quad x \leq c<0, \\
P(X \leq x) \geq 1-p(x), \quad x \geq \bar{c}>0 .
\end{gathered}
$$

Substituting $x_{L}=\left(x_{\min }-\mu\right) / \sigma \leq c<0$ into the first inequality and $x_{U}=\left(x_{\max }-\mu\right) / \sigma \geq \bar{c}>0$ into the second inequality, one gets the bounds

$$
x_{L} \cdot \sigma \leq x_{i}-\mu \leq x_{U} \cdot \sigma, \quad i=1, \ldots, n,
$$

TABLE 1

\begin{tabular}{lccl}
\hline Condition & $F_{\text {st, } \max }^{(4)}(x)$ & $F_{\text {st, } \min }^{(4)}(x)$ & Extremal support $Z_{x}$ \\
\hline$x \leq c$ & 0 & $p(x)$ & $\{x, \varphi(x, \psi(x)), \psi(x)\}$ \\
$c \leq x \leq \bar{c}$ & $p(\psi(x))$ & $1-p(\varphi(x, \psi(x))$ & $\{\psi(x), x, \varphi(x, \psi(x))\}$ \\
$x \geq \bar{c}$ & $1-p(x)$ & 1 & $\{\varphi(x, \psi(x)), \psi(x), x\}$ \\
\hline
\end{tabular}

where $x_{L} \leq c<0, x_{U} \geq \bar{c}>0$ are both solutions of the equation $p(x)=1 / n$, which by (13) is equivalent to the quartic equation

$$
x^{4}-2 S x^{3}+K x^{2}+2 S x-(n-1) \Delta+1=0 .
$$

Since the probability function $p(x)$ is monotone decreasing, the condition $x_{U} \geq \bar{c}>0$ is equivalent to the inequalities

$$
\begin{aligned}
p\left(x_{U}\right) & =\frac{1}{n} \leq p(\bar{c})=\frac{1}{1+\bar{c}^{2}}=\frac{1}{2}\left(1-\frac{S}{\sqrt{4+S^{2}}}\right) \\
& \Longleftrightarrow n \geq \frac{1}{2}\left(4+S^{2}+S \sqrt{4+S^{2}}\right) .
\end{aligned}
$$

Similarly, the condition $x_{L} \leq c<0$ is equivalent to the inequalities

$$
\begin{aligned}
p\left(x_{L}\right) & =\frac{1}{n} \leq p(c)=\frac{1}{1+c^{2}}=\frac{1}{2}\left(1+\frac{S}{\sqrt{4+S^{2}}}\right) \\
& \Longleftrightarrow n \geq \frac{1}{2}\left(4+S^{2}-S \sqrt{4+S^{2}}\right) .
\end{aligned}
$$

Therefore, a necessary condition for the validity of (15) is $n \geq$ $(1 / 2)\left(4+S^{2}+|S| \sqrt{4+S^{2}}\right)$. The following main result has been shown.

Theorem 3 (generalized Laguerre-Samuelson inequality). Let $x_{1}, x_{2}, \ldots, x_{n}$ be $n$ real numbers with mean $\mu$, standard deviation $\sigma$, skewness $S$, and kurtosis $K$ as defined in Section 1. Suppose that $n \geq(1 / 2)\left(4+S^{2}+|S| \sqrt{4+S^{2}}\right)$ and that quartic equation (16) has two real solutions $x_{L}=x_{L}(S, K, n)<0$ and $x_{U}=x_{U}(S, K, n)>0$. Then, the following bounds hold:

$$
x_{L} \leq \frac{x_{i}-\mu}{\sigma} \leq x_{U}, \quad i=1, \ldots, n .
$$

It is worthwhile to state separately the special case of "symmetric" sequences with vanishing skewness $S=0$.

Corollary 4 (generalized Laguerre-Samuelson inequality for symmetric sequences). Let $x_{1}, x_{2}, \ldots, x_{n}$ be $n \geq 2$ real numbers with mean $\mu$, standard deviation $\sigma$, skewness $S=0$, and kurtosis $K \geq-2$ as defined in Section 1. Then, the following bound holds:

$$
\left|\frac{x_{i}-\mu}{\sigma}\right| \leq \frac{\sqrt{2}}{2} \sqrt{\sqrt{(K+2)(K+4 n-6)}-K}, \quad i=1, \ldots, n .
$$

To illustrate the obtained results consider "completely symmetric" sequences of length $n=2 m \geq 2$ of the type

$$
-x_{m} \leq \cdots \leq-x_{2} \leq-x_{1}<0<x_{1} \leq x_{2} \leq \cdots \leq x_{m},
$$

for which $s_{1}=s_{3}=0$; hence $S=0, K=\left(s_{4} / s_{2}^{2}\right)-3$, and Corollary 4 applies. 
TABLE 2: Simulation of completely symmetric sequences.

\begin{tabular}{|c|c|c|c|c|c|}
\hline \multirow[b]{2}{*}{$n$} & \multicolumn{4}{|c|}{ Normally distributed sequence } & \multirow[b]{2}{*}{$\sqrt{n-1}$} \\
\hline & K & $\max \left|\frac{x_{i}-\mu}{\sigma}\right|$ & Bound (20) & $\sqrt[4]{2 n-3}$ & \\
\hline $10^{2}$ & 0.56186 & 2.93964 & 3.95167 & 3.74642 & 9.94987 \\
\hline $10^{3}$ & -0.25589 & 3.43710 & 6.46976 & 6.68489 & 31.60696 \\
\hline $10^{4}$ & -0.02554 & 3.27371 & 11.85401 & 11.89163 & 99.995 \\
\hline $10^{5}$ & 0.00311 & 4.54175 & 21.15554 & 21.14735 & 316.22618 \\
\hline $10^{6}$ & 0.00119 & 5.19235 & 37.61159 & 37.60602 & 999.9995 \\
\hline \multicolumn{6}{|c|}{ Symmetrized gamma distributed sequence } \\
\hline$n$ & $K$ & $\max \left|\frac{x_{i}-\mu}{\sigma}\right|$ & Bound (20) & $\sqrt[4]{5 n-4}$ & $\sqrt{n-1}$ \\
\hline $10^{2}$ & 3.21672 & 3.75302 & 4.59915 & 4.71922 & 9.94987 \\
\hline $10^{3}$ & 5.20859 & 5.71697 & 9.07142 & 8.40728 & 31.60696 \\
\hline $10^{4}$ & 2.62489 & 5.97893 & 14.61965 & 14.95319 & 99.995 \\
\hline $10^{5}$ & 2.92086 & 7.16258 & 26.45799 & 26.59143 & 316.22618 \\
\hline $10^{6}$ & 2.90432 & 8.36365 & 47.04378 & 47.28707 & 999.9995 \\
\hline \multicolumn{6}{|c|}{ Cauchy distributed sequence } \\
\hline$n$ & $K$ & $\max \left|\frac{x_{i}-\mu}{\sigma}\right|$ & Bound (20) & n. a. & $\sqrt{n-1}$ \\
\hline $10^{2}$ & 8.05412 & 4.62977 & 5.26901 & & 9.94987 \\
\hline $10^{3}$ & 495.87198 & 22.34806 & 22.35226 & & 31.60696 \\
\hline $10^{4}$ & 1948.05208 & 54.49966 & 59.56377 & & 99.995 \\
\hline $10^{5}$ & 47796.85784 & 221.09459 & 221.92773 & & 316.22618 \\
\hline $10^{6}$ & 346611.18426 & 644.25589 & 663.63445 & & 999.9995 \\
\hline
\end{tabular}

Example 5 (normally distributed completely symmetric sequence). Suppose that sequence (21) is independent and identically standard normally distributed with sample kurtosis $K=\left(s_{4} / s_{2}^{2}\right)-3 \approx 0$. Then, one has approximately

$$
\begin{aligned}
\left|x_{i}\right| \leq \frac{\sqrt{2}}{2} \sqrt{\sqrt{(K+2)(K+4 n-6)}-K} & \approx \sqrt[4]{2 n-3}, \\
i & =1, \ldots, n .
\end{aligned}
$$

Example 6 (completely symmetrized gamma distributed sequence). Suppose that the independent and identically symmetrized $x_{i}^{\prime} s \sim \Gamma(\alpha, \beta)$ stem from a gamma distributed sequence. For a completely symmetric sequence (21) of this type the theoretical kurtosis is $K^{\prime}=(\alpha+2)(\alpha+3) / \alpha(\alpha+1)-3$. For example, if $\alpha=1$ one has $K^{\prime}=3$, and Corollary 4 implies the following approximate (theoretical) bound:

$$
\begin{array}{r}
\left|\frac{x_{i}-\mu}{\sigma}\right| \leq \frac{\sqrt{2}}{2} \sqrt{\sqrt{\left(K^{\prime}+2\right)\left(K^{\prime}+4 n-6\right)}-K^{\prime}} \leq \sqrt[4]{5 n-4} \\
i=1, \ldots, n
\end{array}
$$

Example 7 (cauchy distributed completely symmetric sequence). Suppose that the independent and identically symmetrized $x_{i}^{\prime} s$ in (21) stem from a Cauchy distributed sequence with density $f(x)=1 / \pi\left(1+x^{2}\right)$. Though the moments of the Cauchy distribution and the corresponding theoretical bound (20) do not exist, the sample based improved Laguerre-Samuelson bound with $K=\left(s_{4} / s_{2}^{2}\right)-3$ remains valid. Table 2 illustrates numerically.
Table 2 illustrates numerically for some typical simulations of completely symmetric sequences. They all show a substantial improvement over the Laguerre-Samuelson inequality. Moreover, Example 7 of completely symmetric sequences from a Cauchy distribution shows that the improved bound can almost be attained for some random sequences. Moreover, simulations for Examples 5 and 6 suggest that further improvement is possible. According to Hürlimann [5], Theorem IV.2.1, the Chebyshev-Markov extremal distributions with known higher order moments predict still better though even more complex bounds.

\section{Conflict of Interests}

The author declares that there is no conflict of interests regarding the publication of this paper.

\section{Acknowledgment}

The author is grateful to a referee for pointing out a minor error.

\section{References}

[1] S. T. Jensen and G. P. H. Styan, "Some comments and a bibliography on the Laguerre-Samuelson inequality with extensions and applications in statistics and matrix theory," in Analytic and Geometric Inequalities and Applications, T. M. Rassias and H. M. Srivastava, Eds., Kluwer Academic Publishers, Dordrecht, The Netherlands, 1999. 
[2] P. A. Samuelson, "How deviant can you be?" Journal of the American Statistical Association, vol. 63, pp. 1522-1525, 1968.

[3] B. C. Arnold and N. Balakrishnan, Relations, Bounds and Approximations for Order Statistics, vol. 53 of Lecture Notes in Statistics, Springer, New York, NY, USA, 1989.

[4] W. P. Smith, "Letter quoted in 'editor's note"' The American Statistician, vol. 34, p. 251, 1980.

[5] W. Hürlimann, "Extremal moment methods and stochastic orders-application in actuarial science," in BAMV-Boletín de la Asociación Matemática Venezolana, vol. 15 of Research Monograph no. 2, pp. 153-301, 2008.

[6] W. Hürlimann, "Best bounds for expected financial payoffs. I. Algorithmic evaluation," Journal of Computational and Applied Mathematics, vol. 82, no. 1-2, pp. 199-212, 1997.

[7] W. Hürlimann, "Best bounds for expected financial payoffs II: applications," Journal of Computational and Applied Mathematics, vol. 82, no. 1-2, pp. 213-227, 1997.

[8] W. Hürlimann, "Analytical bounds for two value-at-risk functionals," ASTIN Bulletin, vol. 32, no. 2, pp. 235-265, 2002.

[9] K. Pearson, "Mathematical contributions to the theory of evolution. XIX. second supplement to a memoir on Skew variation," Philosophical Transactions of the Royal Society of London A, vol. 216, p. 432, 1916.

[10] J. E. Wilkins, "A note on skewness and kurtosis," Annals of Mathematical Statistics, vol. 15, pp. 333-335, 1944.

[11] V. Guiard, Robustheit I. Probleme der Angewandten Statistik, FZ für Tierproduktion Dummerstorf-Rostock, Berlin, Germany, 4th edition, 1980. 


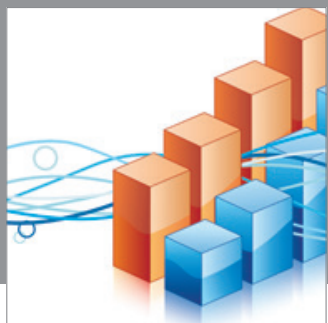

Advances in

Operations Research

mansans

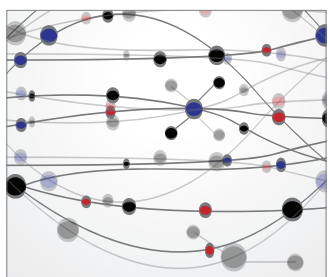

The Scientific World Journal
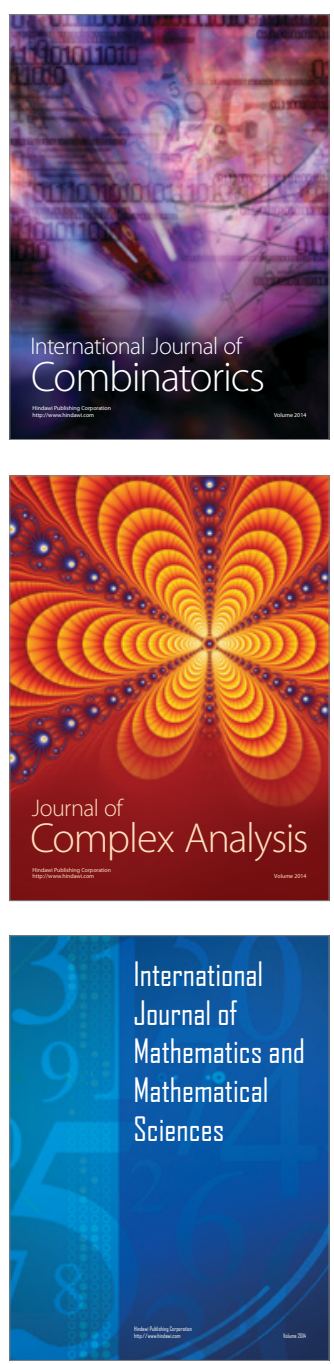
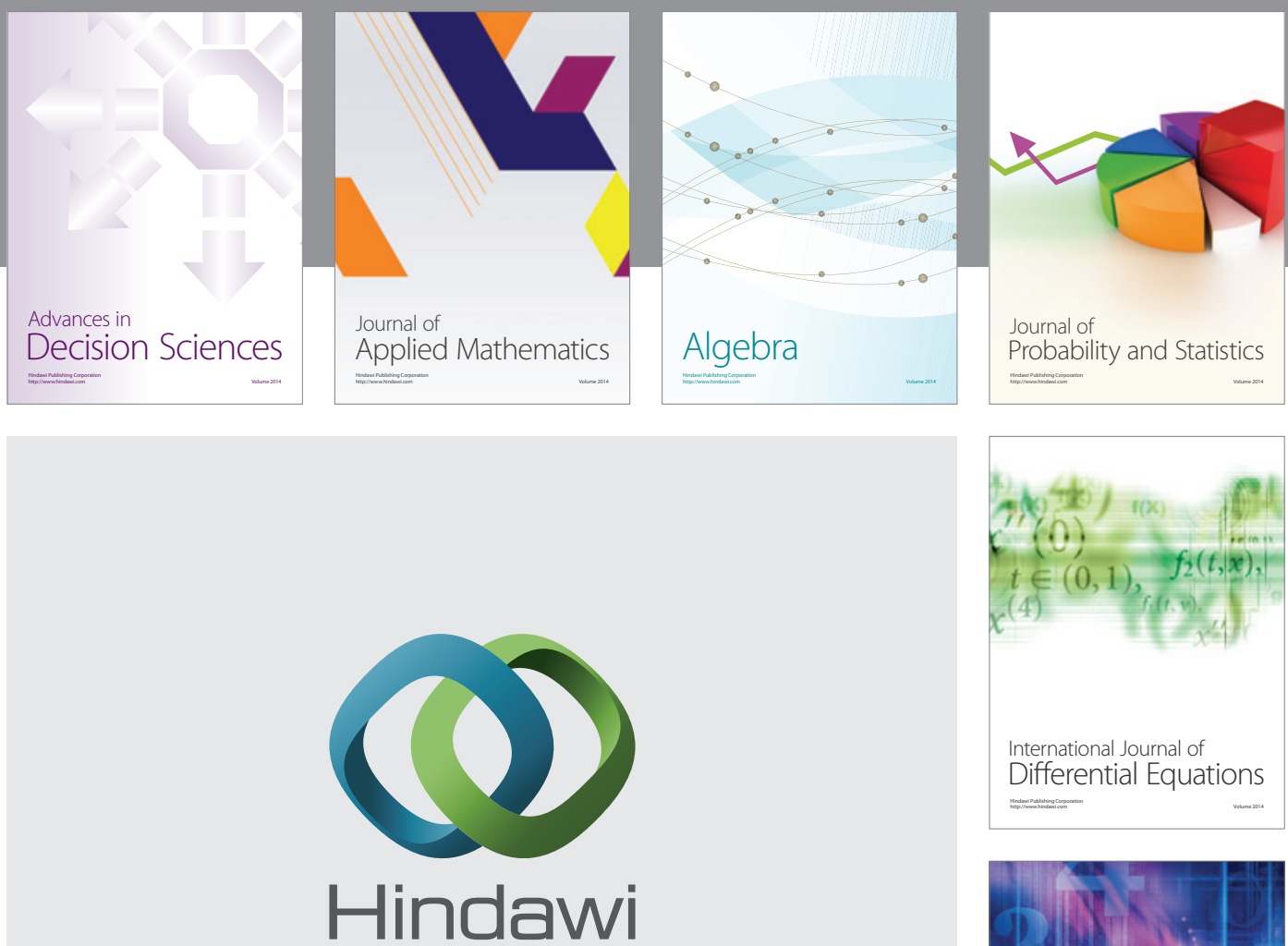

Submit your manuscripts at http://www.hindawi.com
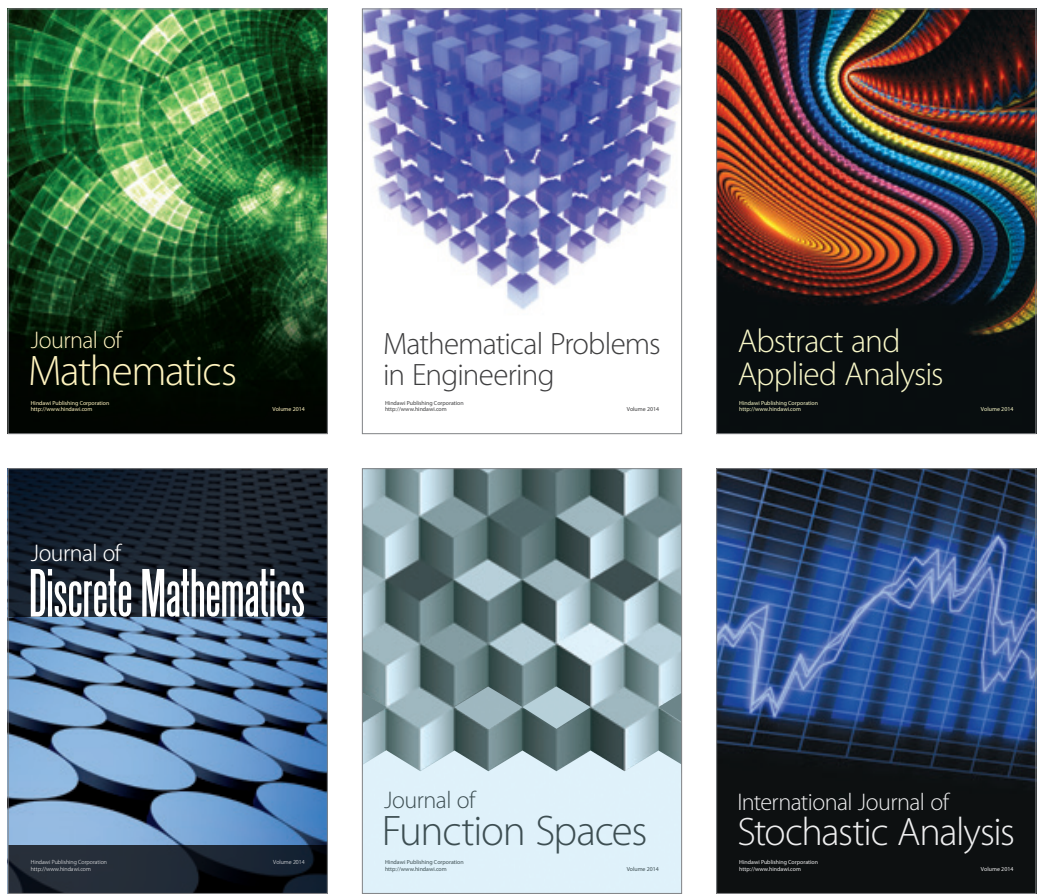

Journal of

Function Spaces

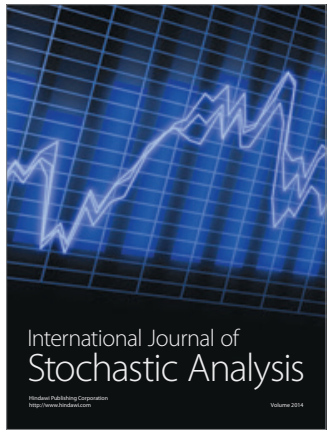

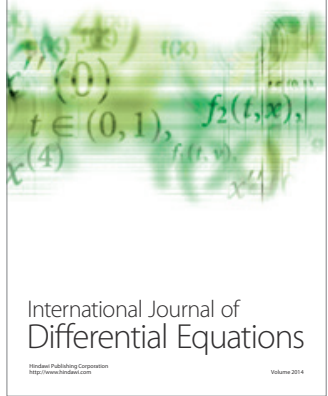
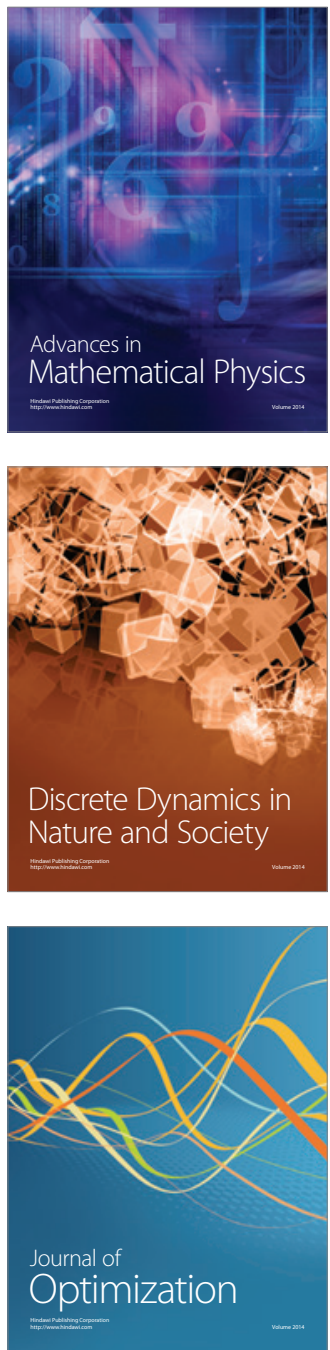